\title{
Metastasizing Ameloblastoma
}

National Cancer Institute

\section{Source}

National Cancer Institute. Metastasizing Ameloblastoma. NCI Thesaurus. Code C54297.

A rare, well differentiated, cytologically benign ameloblastoma which paradoxically metastasizes. 